\title{
RF Sheath-Enhanced Plasma Surface Interaction Studies using Beryllium Optical Emission Spectroscopy in JET ITER-Like Wall
}

\author{
C.Christopher Klepper ${ }^{\mathrm{a}, *}$, Laurent Colas ${ }^{\mathrm{b}}$, Alena Křivskác $^{\mathrm{c}}$, Volodymyr Bobkov ${ }^{\mathrm{d}}$, Philippe Jacquet ${ }^{\mathrm{e}}$, Ephrem Delabie ${ }^{\mathrm{a}}$, \\ Carine Giroud ${ }^{\mathrm{e}}$, Krassimir Kirov ${ }^{\mathrm{e}}$, Ane Lasa ${ }^{\mathrm{a}}$, Ernesto Lerche ${ }^{\mathrm{c}, e}$, Pierre Dumortier ${ }^{\mathrm{c}}$, Frederic Durodié ${ }^{\mathrm{c}}$, Mehdi Firdaouss ${ }^{\mathrm{b}}$ \\ and JET contributors ${ }^{\mathrm{f}}$ \\ ${ }^{a}$ Oak Ridge National Laboratory, Oak Ridge, Tennessee 37831-6169, USA \\ ${ }^{\mathrm{b}}$ IRFM CEA Cadarache 13108 Saint Paul lez Durance France \\ ${ }^{c}$ LPP-ERM/KMS, Royal Military Academy, 30 Avenue de la Renaissance B-1000, Brussels, Belgium \\ ${ }^{\mathrm{d}}$ Max-Planck-Institut für Plasmaphysik, D-85748 Garching, Germany \\ ${ }^{\mathrm{e}}$ CCFE, Culham Science Centre, Abingdon, Oxfordshire OX14 3DB, UK \\ f See the author list of "Overview of the JET results in support to ITER" by X. Litaudon et al. to be \\ published in NF Special issue: overview and summary reports from the 26th Fusion Energy Conf. (Kyoto, \\ Japan, 17-22 Oct. 2016)
}

\begin{abstract}
A dedicated study on JET-ILW, deploying two types of ICRH antennas and spectroscopic observation spots at two outboard, beryllium limiters, has provided insight on long-range (up to 6m) RFenhanced plasma-surface interactions (RF-PSI) due to near-antenna electric fields. To aid in the interpretation of optical emission measurements of these effects, the antenna near-fields are computed using the TOPICA code, specifically run for the ITER-like antenna (ILA); similar modelling already existed for the standard JET antennas (A2). In the experiment, both antennas were operated in current drive mode, as RF-PSI tends to be higher in this phasing and at similar power $(\sim 0.5 \mathrm{MW})$. When sweeping the edge magnetic field pitch angle, peaked RF-PSI effects, in the form of 2-4 fold increase in the local Be source, are consistently measured with the observation spots magnetically connect to regions of TOPICAL-calculated high near-fields, particularly at the near-antenna limiters. It is also found that similar RF-PSI effects are produced by the two types of antenna on similarly distant limiters. Although this mapping of calculated near-fields to enhanced RF-PSI gives only qualitative interpretion of the data, the present dataset is expected to provide a sound experimental basis for emerging RF sheath simulation model validation.
\end{abstract}

\section{Introduction}

Optical emission spectroscopy (OES) is used on JET ITER-Like Wall (JET-ILW) to non-intrusively study RF sheath-enhanced plasma-surface interactions (RF-PSI) in ICRF heated discharges. Such effects are observed on beryllium $(\mathrm{Be})$ outboard limiters, in the form of poloidally-localized regions of enhanced (by $\sim 2-4 x$ ) Be I and Be II light emission. RF-PSIs are also of interest for the study of RF-sheath formation and for the potential impact of localized erosion on ILW component lifetimes. In the early stages of JET-ILW (2011-12) dedicated JET pulses, combining sequential toggling of all available ICRH antennas with concurrent magnetic pitch-angle sweeping, indicated that these RF-PSIs can be traced, via magnetic field-line connection, to regions of anticipated high $\mathrm{E}_{/ /}$near-field at an active antenna $[\mathbf{1}$ - 3]. Such effects are understood as arising from local rectification of these fluctuating near-fields by the plasma near the local limiters, with the resulting potential acting then on the observed (remote) limiter via magnetic connection. Erosion modeling showed that the enhanced optical emission was consistent with increased local erosion of the Be limiter surface $[3,4]$.
In addition to the standard type-"A2" antennas [5], available in the earlier study, the ITER-Like Antenna (ILA) $[6,7]$ has been recently recommissioned $[8]$ and included in a set of new dedicated RF-PSI experiments in the JET 2015-16 Campaign. The aim of this paper is to provide first analyses of the data obtained from the new experimental sessions, with emphasis on the correlation between the optical emission amplification at the observation points, which now include points on a limiter near the ILA, to regions of high parallel components of the near-field $\left(\mathbf{E}_{/ /}\right)$at the active, magnetically connected antenna. Furthemore, we seek to determine whether the RF-PSI induced by the ILA is qualitatively comparable to that induced by the type"A2" antennas for similar phasing and connection geometries. For the type "A2" antennas, the observed, enhanced, long-range RF-PSI effect was shown to be consistent with $\mathrm{E}_{/ /}$near-field A2-specific antenna modeling (TOPICA [9] maps of the active antennas as in Ref. [3]). For the present work, ILA-specific Topica modeling is carried out to see if these correlations also extend to the ILA.

This paper is arranged as follows: In Section 2 we briefly describe the experimental arrangement and cast it 
in the framework of improved experimental capability over the earlier study; in the same section some of the observed responses of the spectroscopic signals to the antenna toggling and simultaneous of the edge safety factor $\left(\mathrm{q}_{95}\right)$ scanning (to change the magnetic connection geometry) are presented. In Section 3, we discuss the magnetic field mapping and then show graphically some of the obtained correlations between the response of the spectroscopic signals and the mapping of these effects to the TOPICA $\mathbf{E}_{/ /}$maps. In Section 4, we summarize and further discuss the findings of these first analyses and the envisioned next steps.

\subsection{Experimental arrangement and approach}

The overall approach to this experiment is similar to that described in Refs $[1,3]$. The principal main differences were in (a) the presence of the ILA and (b) the optical access to an additional poloidal limiter. Figure 1 recalls the toroidal arrangement of the JET standard type-"A2" antennas, as well as the location of the ILA. Furthermore, the Figure shows (in cartoon form - not in exact scale) the location of the observation spots available for these newer measurements. Of most interest is the new access to observation spot "K14", which is nearly centered on a limiter near the ILA.

Observation spot "D14", which was also used in the earlier published studies, was available again with an improvement: As a result of periscope realignment, this spot was nearly centered onto the limiter to the last of
Ant-D, thus sampling the leading edge, where PSI is expected to be peaked. It is recalled that, for the earlier study, this same sightline was shifted to the right, in the Figure, such that its footprint was cast onto the left-most region Ant-D's the recessed Faraday screen. As such, this sightline (coming tangentially fro the left side) would glance by the antenna side of the limiter. This made the interpretation of the measurement challenging in the limiter surface erosion effort of Refs $[3,4]$. The shift of this spot back onto the limiter will also be of great value to the erosion modeling of this newer set of data.

As in the earlier study, all the available antennas were toggled repeatedly, while sweeping the edge safety factor, $\mathrm{q}_{95}$, to vary the magnetic connection between the active antennas and the observation spots. In the present study, the active antennas were Ant-D, Ant-A\&B (i.e. Ant-A and Ant-B, cobined as one) and the ILA.

Unfortunately, Ant-C, whose high $\mathbf{E}_{/ /}$upper corner nearfield cast the most notable bright emission spots onto the central region of Ant-D's left limiter in the earlier study, was not available for the present study. Making up for this, the present access to the "K14" spot provided a measurement point that is nearly equidistant from Ant$\mathrm{D}$ as is "D14" from the ILA, allowing for a quantitative comparison of near-field effect on a distant limiter from each these two very different antenna technologies.

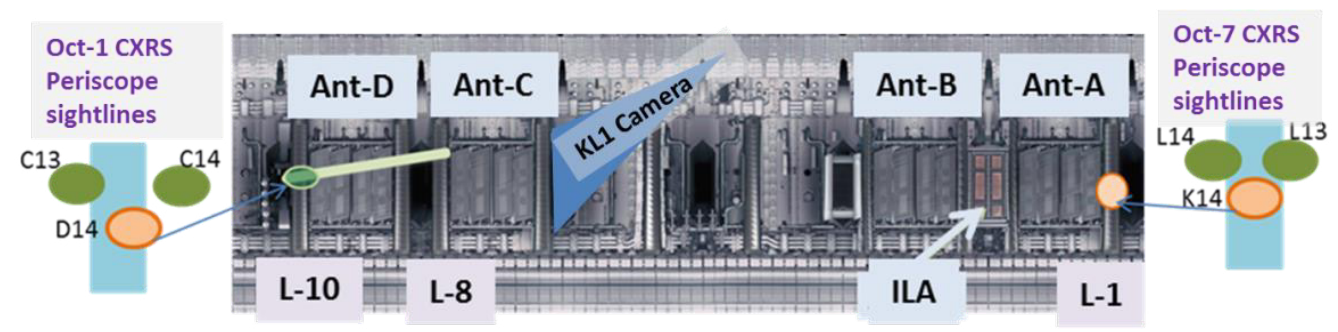

Fig. 1. Position of the new sightline footprints (or spots) on the plasma-facing components of interest, with respect to the position of all JET ICRH antennas in the machine at the time of the Experiment. The spots are named using conventions followed by the JET CXRS diagnostic, whose spectrometers and sightlines are used here. The outboard limiters are arbitrarily labelled for this narrative from L-1 to L-12 (not all seen here) starting with the one on the left of Ant-A, which is in JET octant 1 and going in the direction of increasing octant number.

\section{Spectroscopic detection on antenna- specific PSI}

Figure 2 shows the effect of the toggling of antennas on the local Be spectral line emission at the observation spots D14 and K14 (roughly limiter centered and midplanar) as a function of $\mathrm{q}_{95}$. It is important to note that, although for the pulse of this figure the measurement for both these spots was set for singly ionized beryllium (Be II), they were not using the same spectral line. Thus, the difference in absolute intensities is not of interest in this discussion. (It will become of interest in the upcoming surface erosion modeling effort, in which atomic physics models of the specific spectral lines are also included).
Of more interest in the present discussion is that the response to the antenna toggling is quite different at the two observation spots, as well as strongly dependent on which antenna is ON.

It is further noted that the local line brightness response to $\mathrm{q}_{95}$ can be substantially different than that seen in the equivalent graph of Ref. [1]. In the latter, the effect of switching on Ant-C on the brightness at the observed (left-side) limiter of Ant-D had a minimum in the middle of the $\mathrm{q}_{95}$ scan. In contrast, for the present data, the impact of switching on the ILA on (essentially the same) spot "D14" exhibits continuous monotonic and substantial increase as a function of $\mathrm{q}_{95}$. In the same sweep, the effect of Ant-D on this same spot shows 
much less variation and, if anything, it has maximum rather than a minimum in the middle of the sweep.

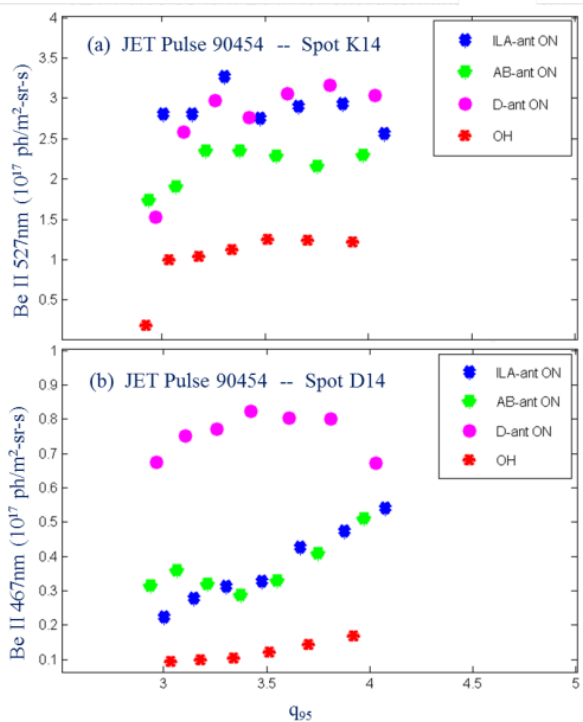

Fig. 2. Plateau-averages of the response of the spectral emission at the two principal spot location from Fig. 2, now plotted separately by active antenna and as a function of $\mathrm{q}_{95}$.

As with the earlier data set, camera imaging could be used to confirm that the spot measurements indeed correspond to poloidally-localized phenomena. In this new experiment, one of the Be II-filtered, wide angle cameras provided discernable data, albeit for a different limiter than for the spot measurements (Fig. 1).

An attempt to understand some of the patterns of Fig. 2 will be made in the next section with magnetic field and $\mathbf{E}_{/ /}$mapping. Meanwhile, it is important also to emphasize that almost all these data sets show a significant enhancement in the local Be emission. To see this, one must compare each point to the corresponding Ohmic phase level (the red points in both graphs). Even in the absence of absolute Be influx values, the relative change in brightness, from Ohmic to Ant-(A\&B or D or ILA) ON is valid for quantitative comparison between the two observation spots.

\section{Qualitative interpretation via magnetic field tracing to active antenna $E_{/ /}$near- field mapping with TOPICA}

Topica $\mathbf{E}_{/ /}$maps of the "A2" antennas, for conditions similar to the ones used in this new experiment, have been previously presented $[3,10]$. For the interpretation of the present experiment, Topica modeling was carried out for the ILA in the three configurations in which it was possible to operate it for this study: Bottom half only, top half only, full antenna, and also for both dipole and current drive phasings. A detailed description of this modeling will be available in Ref [11].

For analysis intended in this paper, the TOPICA $\mathbf{E}_{/ /}$ mappings were calculated for a plane located $5 \mathrm{~mm}$ beyond the near-ILA poloidal limiters. This was done because it is desired here to make correlations of the measured line emission brightness to $\mathbf{E}_{/ /}$variations at the limiters near the active antenna, rather than at the recessed antenna surface. Additional $\mathbf{E}_{/ /}$mappings were calculated Ref [11] for a plane located close to the recessed surface (and thus in the antenna private region) because the aim is ultimately to model the propagation of the RF sheath effects around the limiters (and out of the private region) using a simulation of RF sheath formation and propagation in the SOL for a refined (and potentially extrapolable) interpretation of the experimental measurements [12].

For mapping the magnetic field connections, a code, LHconlen, initially developed to study the impact of the ICRH antennae on LHCD coupling [13] was deployed for the present study, with small modifications. In addition, a custom version of the PFCFlux code [14] was also deployed for this study, partly to provide a check of the 2D maps from the first approach, by providing a special output containing the coordinated at which the fieldlines, emanating from the observation spots, pass over the leading edge of the active antenna's limiters.
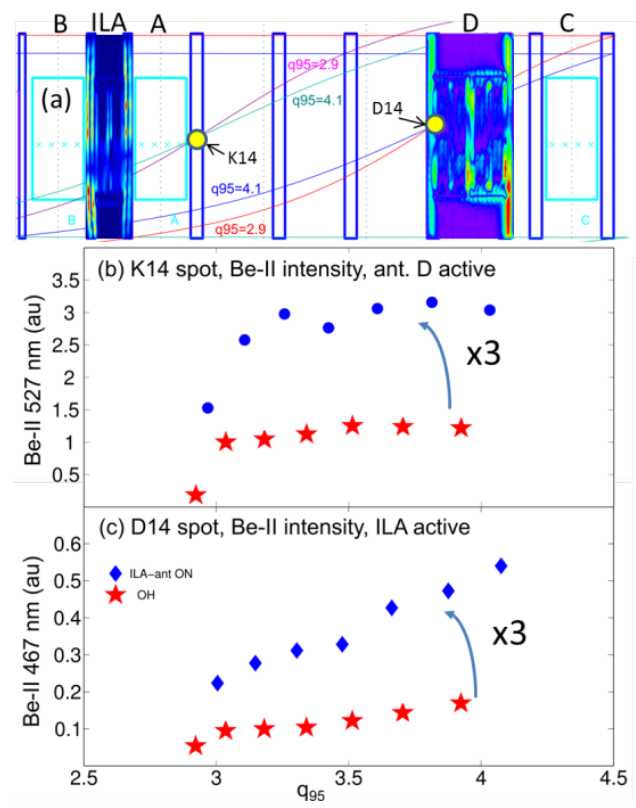

Fig. 3. (a) Magnetic connections for spots K14, D14 to similarly distant antennas: Type-“A2" and ILA, correspondingly. (b, c) Enhanced Be flux comparison, Type"A2" Ant-D to ILA, at the corresponding, distant antenna points.

Figure 3 shows the magnetic connection the distant spot D14 to the ILA and the, quite similar, connection from the distant spot K14 to the Type "A2" Ant-D. It is noted that, in both cases, the measurement spot samples (via the $\mathrm{q}_{95}$ sweep of the magnetic connection) a limited range in the very edge of the corresponding antenna limiter, as well as outside the poloidal range of the main antenna structure. However, looking also at the superimposed and corresponding Topica $\mathbf{E}_{/ /}$maps, it is seen that significant $\mathbf{E}_{/ /}$nearfields are indeed exhibited also at these extreme edges. 
In the same Figure, the response of local Be emission for both cases are shown for all $\mathrm{q}_{95}$ values of the antenna toggling for the same pulse as in Fig. 2. The two important features of these responses are: (1) they both decrease to nearly the Ohmic level when the magnetic fieldline connection gets beyond the poloidal range of the limiter(s) and (2) the relative increase in the emission (over Ohmic) is similar for the two different antenna technologies. Thus, with this observation, this new dataset confirms the previously published interpretation of the 2011-2012 campaign effect, in which rectification of near-fields at the distant antenna leads to the observed Be flux amplification factor (in principle corresponding to a similar local surface erosion enhancement factor) via the magnetic connection. This same effect is now observed at roughly twice the distance along the fieldlines than there was in the earlier study (i.e. the effect of Ant-C on the Ant-D's left limiter, in the connection indicated by the short diagnonal line in Fig. 1). It is now observed arising also from ILA, an antenna technology that is substrantially different than that of JET's standard "A2" antennas [15].

The vanishing of the amplification factor when the magnetic connection goes off the limiter helps further buttress the interpretation that these RF-PSI phenomena being studied with this RF-specific Be spectroscopy method are indeed resulting for these long-range effects (via magnetic connection) from near-field rectification at the antenna and its near-limiters. If they were resulting from far fields forming local sheaths at the observation spots, it would be difficult to explain this transition of the measured effect at the end of the poloidal range of the antenna limiter.

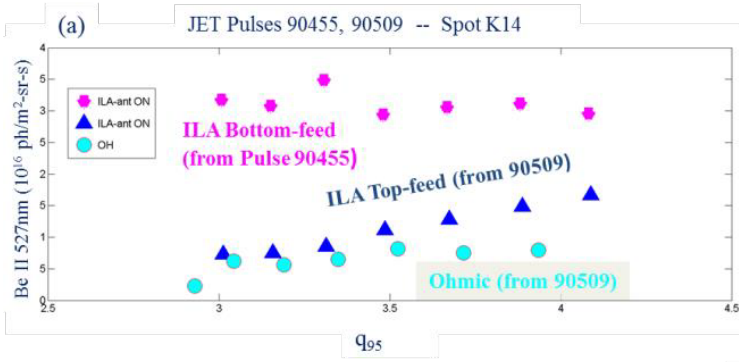

(b) TOPICA-ILA, Top-Feed $[0,-\pi / 2]$
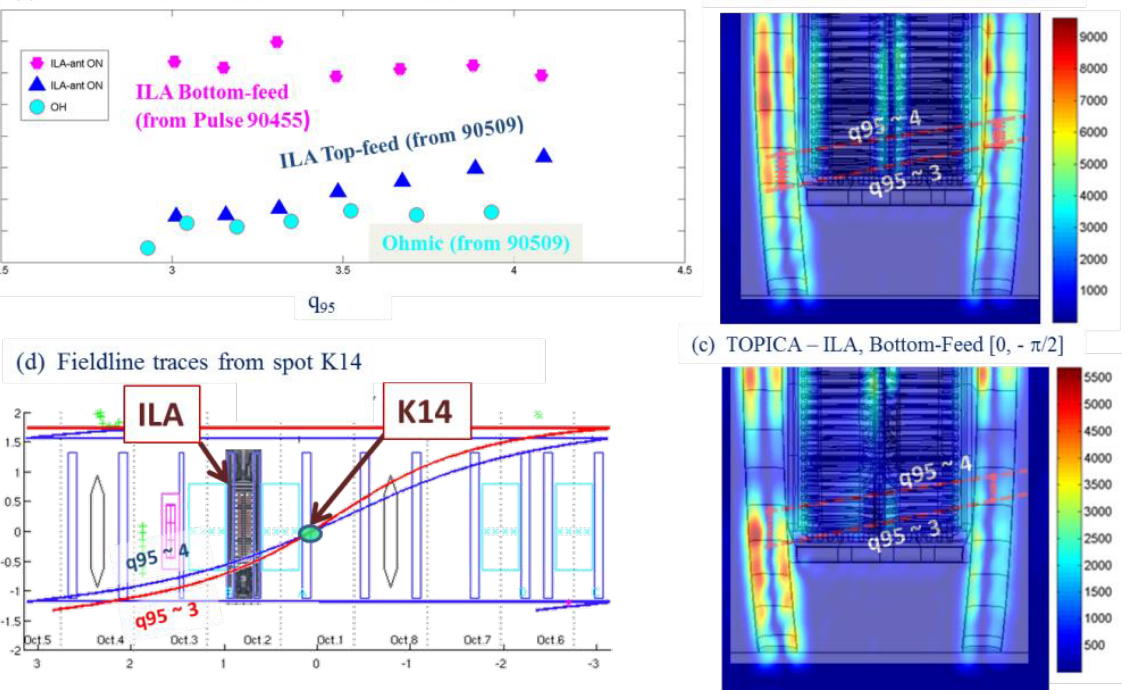

Fig. 4. Response of local Be emission at spot K14 to the ILA, including a comparison between two ILA operation configurations in similar pulses. (a) Plateau-averages of emission at the near ILA K14 spot location for the two configurations and as a function of $\mathrm{q}_{95}$. (b, c) Upper and lower, correspondingly, feed configuration $\mathbf{E}_{/ /}$maps obtained with TOPICA for the current drive [0, $\left.-\pi / 2\right]$ phsing used in the pulse sequence. (d) Corresponding fieldline tracing, showing only extremes $q=\sim 3$ and $q=\sim 4$. The red "*” marks on top of the $\mathbf{E}_{/ /}$maps are the locations where seven fieldlines for the $\mathrm{q}_{95}$ values within this range cross the leading edge of each of the two limiters of the ILA. These at the same $\mathrm{q}_{95}$ values as in the graphs of Be II versus $\mathrm{q}_{95}$.

Figure 4 pertains to the response to the ILA of the local Be emission at spot K14 (relatively close to the ILA) and including a comparison between two ILA operational configurations: only top and only bottom half of the antenna being fed by the generator. It can be seen that, in the bottom-feed case (lower $\mathbf{E}_{/ /}$image) the K14emanating fieldlines "sample" a roughly uniform, strong $\mathbf{E}_{/ /}$region on the left-side limiter of the ILA, while missing any significant $\mathbf{E}_{/ /}$on the right-side. This is consistent with a nearly flat Be II response, which is nevertheless substantially amplified over Ohmic (also plotted in the Figure).

On the other hand, in the upper-antenna-feed case (upper $\mathbf{E}_{/ /}$image), the K14-emanating fieldlines now sample regions of lower $\mathbf{E}_{/ /}$, with respect to the maximum for this calculation (i.e. in the images shown the values are autoscaled to the maximum value for each case). However, although operated at about the same input power $(\sim 0.5 \mathrm{MW})$ the TOPICA calculation yields a much higher ( $\sim 2$-fold) maximum $\mathbf{E}_{/ /}$for the top-feed case and this leads to non-proportional scaling between the $\mathrm{Be}$ emission values and the $\mathbf{E}_{/ /}$values that the nea-antenna limiter regions to which they connect. Curiously, TOPICA calculations in dipole phasing (not shown here) show a substantially decrease in $\mathbf{E}_{/ /}$in the lower part of the antenna for the top-feed case, in better agreement with the experiment (which was, however, carried out in current-drive phasing). This might be considered as an indication of the limits to using antenna near-field maps to interprete the observed $\mathrm{Be}$ emission $\mathrm{q}_{95}$ profiles and 
additional motivation to pursue a full RF sheath simulation as discussed in $[11,12]$.

\section{Summary and Conclusions}

This newer, JET 2015-16 campaign experiment, followup to the 2011-12 RF-PWI study in an ILW environment, further confirms to potential of strong $\mathbf{E}_{/ /}$ near-fields to induce localized impurity sources at distant plasma-facing components, up to several meters from the active antenna, via magnetic connection. This newer dataset also included the ILA, additional observation spots, as well as larger distances on magnetic connection between active antenna and observed RF-PSI location. Local Be flux enhancement factors induced by the ILA were found to be similar to those of an "A2" antenna (at similar antenna to observed PFC distance).

A more detailed interpretation of this new dataset was sought using new antenna modeling results produced specifically for the ILA using TOPICA. Although the range of poloidal extent sampled along the antenna limiter over the available range of the $\mathrm{q}_{95}$ scan was quite small, with respect to the significan spatial features of the modeled $\mathrm{q}_{95}$, it was still possible to qualitatively correlate local maxima in the $\mathrm{Be}$ II emission to magnetically connected maxima in the $\mathbf{E}_{/ /}$poloidal distributions on the active antenna's near-limiters. However, quantitative agreement is not reached, further motivating the planned pursuit of a full, RF sheath rectification model, including transport of rectified potentials and currents in the scrape-off later.

In additional to the RF sheath simulation, the surface erosion side of the study is also under way. The availability of additional spectral lines (some simultaneously for different observation spots, e.g. spots L13, L14, on the same limiter as K14, and some on similar pulses, by scanning the tunable spectrometer associated with D14) will be exploited to improve on long pulse-relevant Be component erosion estimates.

This work was supported, in part, by the US DOE under Contract No. DE-AC05-00OR22725 with UT-Battelle, LLC. This work has been carried out within the framework of the EUROfusion Consortium and has received funding from the Euratom research and training programme 2014-2018 under grant agreement No 633053. The views and opinions expressed herein do not necessarily reflect those of the European Commission.

\section{References}

1. C.C. Klepper et al., J. Nucl. Mater. 438, S594-S598 (2013)

2. P. Jacquet et al., Physics of Plasmas (1994-present) 21, 061510 (2014); doi: 10.1063/1.4884354
3. C.C. Klepper et al., Phys. Scripta, vol. 2016, no. T167 (2016) 014035

4. A. Lasa et al., "ERO Modeling and Sensitivity Analysis of Erosion Enhanced by Magnetically Connected Antennas," Submitted to NF (Apr. 2017)

5. A. Kaye, T. Brown, V. Bhatnagar, P. Crawley, et al., Fus. Engin. Design 24, 1 (1994)

6. F. Durodié, at al., "Physics and engineering results obtained with the ion cyclotron range of frequencies ITER-like antenna on JET," Plasma Phys. Control. Fusion 54 (2012) 074012

7. A. Czarnecka at al., Plasma Phys. Control. Fusion 54 (2012) 074013

8. P. Dumortier et al., this conference.

9. D. Milanesio, O. Meneghini, V. Lancellotti, R. Maggiora, and G. Vecchi, Nucl. Fusion 49, 115019 (2009).

10. Vl. Bobkov et al., Journal of Nuclear Materials 438 (2013) S160-S165

11. A. Křivska et al., This Conference

12. L. Colas, et al., Overview Talk in this Conference.

13. K. Kirov et al. PPCF 2009044003

14. M. Firdaouss et al., Journal of Nuclear Materials 438 (2013) S536-S539

15. F. Durodie et al., Operational Experience with the ICRF ITER Like Antenna on JET, EFDA-JETPR(11)48 (EUROfusion Report). 